\title{
Avaliação do Grau de Ansiedade, Depressão e Motivação dos Fumantes que Procuraram Tratamento para Deixar de Fumar no Distrito Federal \\ Evaluation of the Level of Anxiety, Depression and Motivation of Smokers Seeking Treatment for Smoking Cessation in the Federal District \\ Evaluación del Grado de la Ansiedad, de la Depresión y de la Motivación de los Fumadores que Solicitan Tratamiento para Dejar de Fumar en el Distrito Federal
}

\author{
Maria Suélita de Lima', Carlos Alberto de Assis Viegas²
}

\section{Resumo}

Introdução: Ansiedade, depressão e baixa motivação podem interferir no sucesso do tratamento do tabagismo. Objetivo: Avaliar o grau de ansiedade, depressão e motivação dos fumantes atendidos no Distrito Federal. Método: Estudo transversal, envolvendo 1.233 fumantes atendidos em 19 Centros de Referência. Instrumentos utilizados: Hospital Anxiety and Depression Scale, teste de motivação de Richmond e ficha com dados sociodemográficos. Para análise dos dados, foi utilizado, quando pertinente, teste $t$ de Student, qui-quadrado e correlação de Spearman. Resultados: Mulheres apresentaram maiores graus de ansiedade $(\mathrm{p}<0,01)$, depressão $(\mathrm{p}<0,01)$, e de motivação $(\mathrm{p}<0,01)$ para deixar de fumar, quando comparadas com os homens. Não houve diferença entre motivação e as variáveis sociodemográficas $(\mathrm{p}>0,05)$. Baixa renda e baixa escolaridade foram associadas aos níveis de ansiedade $(\mathrm{p}<0,01$ e $\mathrm{p}<0,03)$ e depressão $(\mathrm{p}<0,01$ e $\mathrm{p}<0,04)$. Idade de início do tabagismo foi associada ao nível de depressáo; quanto mais jovem o início do tabagismo, maior probabilidade de depressão $(\mathrm{p}<0,02)$. Não houve correlação entre número de cigarros/dia e grau de motivação para deixar de fumar, com níveis de ansiedade e depressão. A motivação não foi associada aos níveis de ansiedade e depressão. Conclusáo: Houve associação entre os níveis de ansiedade, depressão e tabagismo. Cerca de $30 \%$ dos fumantes apresentaram níveis prováveis de ansiedade e depressão; destes, 50\% tinham motivação elevada para deixar de fumar, principalmente as mulheres. Esses achados apontam para a importância da avaliação desses sintomas e da motivação, no início do tratamento, para elevar as taxas de cessação.

Palavras-chave: Abandono do Uso de Tabaco; Ansiedade; Depressão; Motivação

\footnotetext{
${ }^{1}$ Assistente Social do Núcleo de Prevenção da Secretaria de Estado e Saúde do Distrito Federal. Mestranda do Departamento de Ciências Médicas da Universidade de Brasília (UnB) Brasília (DF), Brasil. E-mail: lima828@gmail.com.

${ }^{2}$ Professor Associado Departamento de Clínica Médica da Faculdade de Medicina da UnB Brasília (DF), Brasil. E-mail: viegasc@uol.com.br.
} 


\section{INTRODUÇÃO}

Apesar de bastante reconhecidos os efeitos nocivos do uso do tabaco, segundo dados do Instituto Brasileiro de Geografia (IBGE), no Brasil, em 2008, o percentual de fumantes foi de $17,5 \%$ entre pessoas de 15 anos ou mais, o que correspondia ao contingente de 25 milhóes de pessoas. No Distrito Federal o percentual de fumantes encontrado foi de $13,4 \%{ }^{1}$.

Entre as razóes que os fumantes relatam para continuar fumando, está o alívio dos sintomas de ansiedade e depressão. Além disso, sabe-se que a ansiedade está associada com risco aumentado de lapso durante a cessaçáo do tabagismo, e os sintomas depressivos são preditores significativos de lapsos e recaídas precoces ${ }^{2}$. Recorda-se que muitas pessoas que preenchem critérios diagnósticos para transtornos mentais não procuram tratamento para essas condiçôes, o que torna importante investigar a relação entre doença mental e tabagismo ${ }^{3}$. Assim, um olhar atento para as questóes relacionadas à ansiedade e depressão de fumantes em tratamento para cessação do tabagismo, bem como a oferta de tratamento concomitante, pode vir a ser uma medida importante no auxílio ao enfrentamento da abstinência à nicotina ${ }^{4}$.

Outro ponto importante nesse processo é a motivação, que é condição imprescindível para iniciar o tratamento, e sua ausência praticamente elimina as expectativas de abstinência. O conhecimento das características associadas à motivação para deixar de fumar e a interrupção do tabagismo são importantes porque permitem identificar grupos com maior e menor probabilidades de deixar de fumar e, assim, adequar estratégias de abordagem ${ }^{5}$.

Assim, o objetivo deste estudo foi avaliar os graus de ansiedade, depressão e motivação em pacientes que procuram tratamento para deixar de fumar nos Centros de Referência de Tratamento de Tabagismo do Distrito Federal.

\section{MÉTODO}

Foi realizado um estudo transversal descritivo, com 1.233 pacientes atendidos em 19 Centros de Referência de Tratamento de Tabagismo do Distrito Federal. Os dados foram coletados no período de fevereiro a setembro de 2009 por profissionais que realizam o atendimento e que foram previamente capacitados pelos pesquisadores. A pesquisa foi conduzida após aprovação do Comitê de Ética em Pesquisa (CEP) da Fundação de Ensino e Pesquisa em Ciências da Saúde (FEPECS) SES/DF (Carta no 256/09 - CEP/SES), tendo como base a Resolução 196/96 CNS/MS, que dispõe sobre "pesquisa envolvendo seres humanos". Todos os pacientes participantes do estudo assinaram o Termo de Consentimento Livre e Esclarecido e preencheram uma ficha clínica quando ingressaram no programa de cessação tabágica.

As informaçôes sobre os graus de ansiedade e depressão foram medidas com a Escala (HADS) Hospital Anxiety and Depression Scale. Para cada item, pode ser conferido $0,1,2$ ou 3 pontos, em que questóes ímpares avaliam o grau de ansiedade (HADS-A) e pares o grau de depressão (HADS-D). Foram adotados os pontos de corte apontados por Zigmond e Snaith ${ }^{6}$, recomendados para ambas as situaçōes: de 0-7 pontos: improvável, 8-11 pontos: possível (questionável ou duvidoso) e 12-21: provável. No início, a HADS foi desenvolvida para identificar sintomas de ansiedade e depressão em pacientes de hospitais clínicos não psiquiátricos, sendo posteriormente utilizada $\mathrm{em}$ pacientes não internados e sem doenças diagnosticadas ${ }^{7}$. Opta-se por essa escala por ser de fácil manuseio e de rápida execução, podendo ser realizada pelo paciente e pelo entrevistador.

A motivação foi avaliada pelo teste de Richmond, sendo adotados os pontos de corte sugeridos pelo autor ${ }^{8}$ : de 0-6: motivaçáo baixa, 7-9: motivação moderada e 10: motivação elevada. Os dados sociodemográficos foram obtidos a partir da "Ficha Clínica dos Ambulatórios de Tabagismo". Os dados foram analisados por meio do programa Statistical Package for the Social Sciences (SPSS) versão 17.0 , utilizando para comparação das variáveis os testes $t$ de Student ou qui-quadrado e correlação de Spearman, quando pertinente; o nível de significância adotado foi de $5 \%(\mathrm{p} \leq 0,05)$.

\section{RESULTADOS}

Foi pesquisado um total de 1.233 fichas de pacientes provenientes de várias Unidades de Saúde do Distrito Federal. Na Tabela 1, apresentam-se os dados sociodemográficos e econômicos da amostra, em que se pode observar que a maioria dos pacientes, $65 \%$, era do gênero feminino. A idade variou entre 19 e 77 anos, com média de $44 \pm 11$ anos. A idade de início do tabagismo ficou entre 5 e 40 anos, com média de $16 \pm 4,7$ anos; e, em $70 \%$ da amostra, a idade de início do tabagismo ficou abaixo dos 20 anos.

$\mathrm{Na}$ categorização por renda familiar, os pacientes que recebiam menos de 1 salário mínimo (SM) equivalem a 13\%, aqueles com renda de 1 a 2 SM somam $15 \%$, de 2 a 4 SM $10 \%, 4$ a 6 SM - 3\%, 6 a 8 SM - $2 \%$, e de 8 ou mais SM - $4 \%$.

Em relação à escolaridade, nota-se que a maioria dos pacientes pesquisados, $43 \%$, tem nível fundamental, $37 \%$ concluíram o nível médio e pouco mais de $11 \%$ têm nível superior. Foi observado também que $6, \%$ dos pacientes não são alfabetizados. 
Tabela 1. Distribuição da população em relação às variáveis sociodemográficas e econômicas

\begin{tabular}{|c|c|c|c|}
\hline \multicolumn{2}{|c|}{ Fator estudado } & $\mathbf{n}$ & $\%$ \\
\hline \multirow{2}{*}{ Sexo } & Masculino & 427 & 34,6 \\
\hline & Feminino & 806 & 65,4 \\
\hline \multirow{5}{*}{ Faixa etária } & Menos de 30 anos & 165 & 13,4 \\
\hline & 31 a 40 anos & 250 & 20,3 \\
\hline & 41 a 50 anos & 432 & 35 \\
\hline & 51 a 60 anos & 278 & 22,5 \\
\hline & 61 ou mais & 108 & 8,8 \\
\hline \multirow{5}{*}{$\begin{array}{l}\text { Faixa de idade de início } \\
\text { do tabagismo }\end{array}$} & Menos de 10 anos & 98 & 7,9 \\
\hline & 11 a 20 anos & 949 & 77 \\
\hline & 21 a 30 anos & 100 & 8,1 \\
\hline & Mais de 30 anos & 20 & 1,6 \\
\hline & Não informado & 66 & 5,4 \\
\hline \multirow{7}{*}{$\begin{array}{l}\text { Faixa de renda em } \\
\text { salários mínimos }\end{array}$} & Menos de $1 \mathrm{SM}$ & 161 & 13,1 \\
\hline & 1 a 1,99 SM & 190 & 15,4 \\
\hline & 2 a 3,99 SM & 119 & 9,6 \\
\hline & 4 a 5,99 SM & 42 & 3,4 \\
\hline & 6 a 7,99 SM & 27 & 2,2 \\
\hline & 8 ou mais SM & 54 & 4,4 \\
\hline & Não informado & 640 & 51,9 \\
\hline \multirow{5}{*}{ Escolaridade } & Não alfabetizado & 76 & 6,2 \\
\hline & Fundamental & 531 & 43,1 \\
\hline & Médio & 459 & 37,2 \\
\hline & Superior & 143 & 11,6 \\
\hline & Não informado & 24 & 1,9 \\
\hline
\end{tabular}

Na Tabela 2, apresentam-se os níveis de depressão da população estudada e sua distribuição dentro das variáveis analisadas. É possível verificar que as mulheres são, de forma estatisticamente significativa $(\mathrm{p}<0,001)$, mais deprimidas do que os homens e que a chance de uma mulher ser deprimida é três vezes maior em relação ao homem. Nas diferentes faixas etárias, não foram encontradas diferenças estatisticamente significativas quanto à depressão, embora se possa observar uma tendência a ser mais deprimida a população na faixa etária de 41 a 50 anos de idade. A média de idade dos pacientes com depressão provável foi de $45 \pm 12$ anos. Ao comparar a idade de início do tabagismo com o nível de depressão, a relação encontrada foi significativa $(\mathrm{p}=0,025)$, de onde se extrai que os pacientes com nível provável de depressão iniciaram o tabagismo com idade média de $15 \pm 5$ anos.

Quanto à renda familiar, observa-se maior depressão provável nas faixas menos favorecidas de forma estatisticamente significativa $(p=0,001)$. De forma semelhante, os fumantes com menor escolaridade apresentaram significativamente $(\mathrm{p}=0,003)$ maior chance de depressão do que os com maior escolaridade. No tocante à quantidade de tabaco consumida por dia e a escala de depressão, não foi verificada nenhuma associação estatisticamente significativa ( $p>0,05)$.

$\mathrm{Na}$ Tabela 3, observam-se as variáveis em relação ao nível de ansiedade, na qual se pode verificar que o nível de ansiedade entre homens e mulheres apresentou diferença significativa $(\mathrm{p}<0,001)$, e que as mulheres apresentam nível provável de ansiedade três vezes maior do que os homens. Com relação à faixa etária, nota-se que o nível de ansiedade está relacionado com a idade do paciente $(\mathrm{p}=0,008)$. Aqueles com idade entre 41 e 50 anos foram os que mais apresentaram probabilidade de serem ansiosos. Se encontra ainda tendência de associação entre a idade de início do tabagismo e o nível provável de ansiedade $(\mathrm{p}=0,057)$.

Assim como na depressáo, no que se refere à faixa salarial, observa-se maior ansiedade provável nas faixas menos favorecidas de forma estatisticamente significativa 
Tabela 2. Nível de depressão em relação às variáveis sociodemográficas

\begin{tabular}{|c|c|c|c|c|c|c|}
\hline & & \multicolumn{3}{|c|}{ Escala de depressão (HAD) } & \multirow{2}{*}{ Total } & \multirow{3}{*}{$\begin{array}{l}\text { Teste Qui- } \\
\text { quadrado } \\
\text { (p-valor) }\end{array}$} \\
\hline & & Improvável & Possível & Provável & & \\
\hline & & $\mathbf{n}$ & $\mathbf{n}$ & $\mathbf{n}$ & $\mathbf{n}$ & \\
\hline \multirow{3}{*}{ Sexo } & Masculino & 248 & 108 & 60 & 416 & \multirow{3}{*}{0,000} \\
\hline & Feminino & 334 & 237 & 213 & 784 & \\
\hline & Total & 582 & 345 & 273 & 1.200 & \\
\hline \multirow{6}{*}{ Faixa etária } & Menos de 30 anos & 85 & 47 & 30 & 162 & \multirow{6}{*}{0,066} \\
\hline & 31 a 40 anos & 104 & 89 & 52 & 245 & \\
\hline & 41 a 50 anos & 201 & 113 & 106 & 420 & \\
\hline & 51 a 60 anos & 134 & 75 & 59 & 268 & \\
\hline & 61 ou mais & 58 & 21 & 26 & 105 & \\
\hline & Total & 582 & 345 & 273 & 1.200 & \\
\hline \multirow{7}{*}{$\begin{array}{l}\text { Faixa de } \\
\text { renda em } \\
\text { salários } \\
\text { mínimos }\end{array}$} & Menos de $1 \mathrm{SM}$ & 60 & 46 & 52 & 158 & \multirow{7}{*}{0,001} \\
\hline & 1 a $1,99 \mathrm{SM}$ & 86 & 56 & 41 & 183 & \\
\hline & 2 a 3,99 SM & 64 & 29 & 25 & 118 & \\
\hline & 4 a $5,99 \mathrm{SM}$ & 25 & 10 & 6 & 41 & \\
\hline & 6 a $7,99 \mathrm{SM}$ & 18 & 5 & 4 & 27 & \\
\hline & 8 ou mais SM & 37 & 12 & 4 & 53 & \\
\hline & Total & 290 & 158 & 132 & 580 & \\
\hline \multirow{5}{*}{ Escolaridade } & Não alfabetizado & 24 & 27 & 19 & 70 & \multirow{5}{*}{0,003} \\
\hline & Fundamental & 238 & 146 & 136 & 520 & \\
\hline & Médio & 231 & 126 & 92 & 449 & \\
\hline & Superior & 80 & 41 & 18 & 139 & \\
\hline & Total & 573 & 340 & 265 & 1.178 & \\
\hline \multirow{5}{*}{$\begin{array}{l}\text { Faixa de } \\
\text { idade de } \\
\text { início do } \\
\text { tabagismo }\end{array}$} & Menos de 10 anos & 41 & 32 & 22 & 95 & \multirow{5}{*}{0,025} \\
\hline & 11 a 20 anos & 435 & 264 & 225 & 924 & \\
\hline & 21 a 30 anos & 61 & 24 & 12 & 97 & \\
\hline & Mais de 30 anos & 6 & 8 & 6 & 20 & \\
\hline & Total & 543 & 328 & 265 & 1.136 & \\
\hline \multirow{3}{*}{$\begin{array}{l}\text { Quantidade } \\
\text { de tabaco/ } \\
\text { dia }\end{array}$} & $\begin{array}{c}\text { Menos de } 20 \\
\text { cigarros }\end{array}$ & 359 & 204 & 161 & 724 & \multirow{3}{*}{0,519} \\
\hline & $\begin{array}{c}\text { Menos de } 20 \\
\text { cigarros }\end{array}$ & 137 & 93 & 69 & 299 & \\
\hline & Total & 496 & 297 & 230 & 1.023 & \\
\hline
\end{tabular}

$(\mathrm{p}=0,039)$. De forma semelhante, os fumantes com menor escolaridade apresentaram significativamente $(\mathrm{p}=0,001)$ maior chance de ansiedade do que os com maior escolaridade. Em relação à quantidade de tabaco consumida por dia e ansiedade, também não se encontrou nenhuma relação significativa $(\mathrm{p}>0,05)$.

Comparou-se também o grau de motivaçáo dos pacientes em relação às variáveis socioeconômicas e os níveis de ansiedade e depressão. $\mathrm{Na}$ Tabela 4, observa-se que o nível de motivação varia conforme o gênero do paciente. A motivação é diferente entre os homens e as mulheres e essa diferença é significativa $(p=0,006)$. As chances encontradas de um homem ser classificado com baixa motivação é duas vezes maior do que nas mulheres.

A motivação não teve correlação significativa com a faixa etária $(\mathrm{p}=0,607)$; escolaridade $(\mathrm{p}=0,408)$; idade de início do tabagismo $(\mathrm{p}=0,510)$; renda familiar $(\mathrm{p}=0,116)$; quantidade de tabaco consumida por dia $(\mathrm{p}=0,077)$; e as escalas de ansiedade $(\mathrm{p}=0,072)$ e depressão $(\mathrm{p}=0,293)$. 
Tabela 3. Nível de ansiedade em relação às variáveis sociodemográficas

\begin{tabular}{|c|c|c|c|c|c|c|}
\hline & & \multicolumn{3}{|c|}{ Escala de ansiedade (HAD) } & \multirow{2}{*}{ Total } & \multirow{3}{*}{$\begin{array}{l}\text { Teste Qui- } \\
\text { quadrado } \\
\text { (p-valor) }\end{array}$} \\
\hline & & Improvável & Possível & Provável & & \\
\hline & & $\mathbf{n}$ & $\mathbf{n}$ & $\mathbf{n}$ & $\mathbf{n}$ & \\
\hline \multirow{3}{*}{ Sexo } & Masculino & 192 & 130 & 93 & 415 & \multirow{3}{*}{0,000} \\
\hline & Feminino & 192 & 268 & 327 & 787 & \\
\hline & Total & 384 & 398 & 420 & 1.202 & \\
\hline \multirow{6}{*}{ Faixa etária } & Menos de 30 anos & 54 & 59 & 50 & 163 & \multirow{6}{*}{0,008} \\
\hline & 31 a 40 anos & 67 & 76 & 102 & 245 & \\
\hline & 41 a 50 anos & 124 & 146 & 152 & 422 & \\
\hline & 51 a 60 anos & 89 & 90 & 89 & 268 & \\
\hline & 61 ou mais & 50 & 27 & 27 & 104 & \\
\hline & Total & 384 & 398 & 420 & 1.202 & \\
\hline \multirow{7}{*}{$\begin{array}{l}\text { Faixa de } \\
\text { renda em } \\
\text { salários } \\
\text { mínimos }\end{array}$} & Menos de 1 SM & 37 & 58 & 65 & 160 & \multirow{7}{*}{0,039} \\
\hline & 1 a $1,99 \mathrm{SM}$ & 61 & 58 & 64 & 183 & \\
\hline & 2 a 3,99 SM & 33 & 43 & 43 & 119 & \\
\hline & 4 a $5,99 \mathrm{SM}$ & 16 & 13 & 12 & 41 & \\
\hline & 6 a $7,99 \mathrm{SM}$ & 14 & 8 & 5 & 27 & \\
\hline & 8 ou mais SM & 21 & 21 & 10 & 52 & \\
\hline & Total & 182 & 201 & 199 & 582 & \\
\hline \multirow{5}{*}{ Escolaridade } & Não alfabetizado & 16 & 18 & 36 & 70 & \multirow{5}{*}{0,001} \\
\hline & Fundamental & 155 & 167 & 200 & 522 & \\
\hline & Médio & 157 & 148 & 144 & 449 & \\
\hline & Superior & 48 & 58 & 33 & 139 & \\
\hline & Total & 376 & 391 & 413 & 1.180 & \\
\hline \multirow{5}{*}{$\begin{array}{l}\text { Faixa de } \\
\text { idade de } \\
\text { início do } \\
\text { tabagismo }\end{array}$} & Menos de 10 anos & 28 & 26 & 41 & 95 & \multirow{5}{*}{0,057} \\
\hline & 11 a 20 anos & 282 & 317 & 327 & 926 & \\
\hline & 21 a 30 anos & 43 & 28 & 26 & 97 & \\
\hline & Mais de 30 anos & 5 & 9 & 6 & 20 & \\
\hline & Total & 358 & 380 & 400 & 1.138 & \\
\hline \multirow{3}{*}{$\begin{array}{l}\text { Quantidade } \\
\text { de tabaco/ } \\
\text { dia }\end{array}$} & Menos de 20 cigarros & 231 & 252 & 242 & 725 & \multirow{3}{*}{0,253} \\
\hline & Menos de 20 cigarros & 96 & 90 & 114 & 300 & \\
\hline & Total & 327 & 342 & 356 & 1.025 & \\
\hline
\end{tabular}

\section{DISCUSSÃO}

O tabagismo é uma doença de tratamento complexo. A maioria dos fumantes apresenta características peculiares e, muitas vezes, encontram-se populações específicas que demandam atenção diferenciada. Uma avaliação cuidadosa dos pacientes que buscam tratamento é de fundamental importância para se adequar às diversas estratégias de tratamento.

$\mathrm{Na}$ amostra estudada, 59\% dos pacientes deixaram de fumar ao final da quarta sessão do tratamento; $34 \%$ dos pacientes foram classificados com nível provável para ansiedade e $28 \%$ com nível provável para depressão. A motivaçáo foi elevada em $51 \%$ dos casos. No que se refere à ansiedade e depressão, considera-se esse índice alto se compará-lo a outros estudos como o de McClave et al. ${ }^{9}$, que encontraram 14,9\% com diagnóstico de ansiedade e 20,3\% com diagnóstico de depressão. As mulheres apresentaram níveis de ansiedade e depressão maiores do que os homens, dado já constatado por outros autores, cujos achados demonstram quase o dobro em relação aos homens ${ }^{10}$. Outro dado relevante foi o fato de as 
Tabela 4. Nível de ansiedade em relação às variáveis sociodemográficas

\begin{tabular}{|c|c|c|c|c|c|c|}
\hline & & \multicolumn{3}{|c|}{ Motivação (Teste de Richmond) } & \multirow{2}{*}{ Total } & \multirow{3}{*}{$\begin{array}{l}\text { Teste Qui- } \\
\text { quadrado } \\
\text { (p-valor) }\end{array}$} \\
\hline & & Baixa & Moderada & Elevada & & \\
\hline & & $\mathbf{n}$ & $\mathbf{n}$ & $\mathbf{n}$ & $\mathbf{n}$ & \\
\hline \multirow{3}{*}{ Sexo } & Masculino & 32 & 183 & 204 & 419 & \multirow{3}{*}{0,006} \\
\hline & Feminino & 29 & 334 & 426 & 789 & \\
\hline & Total & 61 & 517 & 630 & 1.208 & \\
\hline \multirow{6}{*}{ Faixa etária } & $\begin{array}{c}\text { Menos de } 30 \\
\text { anos }\end{array}$ & 7 & 73 & 82 & 162 & \multirow{6}{*}{0,607} \\
\hline & 31 a 40 anos & 13 & 103 & 129 & 245 & \\
\hline & 41 a 50 anos & 16 & 187 & 223 & 426 & \\
\hline & 51 a 60 anos & 16 & 116 & 138 & 270 & \\
\hline & 61 ou mais & 9 & 38 & 58 & 105 & \\
\hline & Total & 61 & 517 & 630 & 1.208 & \\
\hline \multirow{7}{*}{$\begin{array}{l}\text { Faixa de } \\
\text { renda em } \\
\text { salários } \\
\text { mínimos }\end{array}$} & Menos de $1 \mathrm{SM}$ & 7 & 67 & 86 & 160 & \multirow{7}{*}{0,039} \\
\hline & 1 a $1,99 \mathrm{SM}$ & 8 & 74 & 102 & 184 & \\
\hline & 2 a 3,99 SM & 10 & 53 & 56 & 119 & \\
\hline & 4 a $5,99 \mathrm{SM}$ & 4 & 14 & 23 & 41 & \\
\hline & 6 a $7,99 \mathrm{SM}$ & 3 & 15 & 9 & 27 & \\
\hline & 8 ou mais $S M$ & 3 & 31 & 19 & 53 & \\
\hline & Total & 35 & 254 & 295 & 584 & \\
\hline \multirow{5}{*}{ Escolaridade } & Não alfabetizado & 4 & 32 & 34 & 70 & \multirow{5}{*}{0,408} \\
\hline & Fundamental & 26 & 211 & 283 & 520 & \\
\hline & Médio & 21 & 196 & 236 & 453 & \\
\hline & Superior & 9 & 71 & 61 & 141 & \\
\hline & Total & 60 & 510 & 614 & 1.184 & \\
\hline \multirow{3}{*}{$\begin{array}{l}\text { Quantidade } \\
\text { de tabaco/ } \\
\text { dia }\end{array}$} & $\begin{array}{c}\text { Menos de } 20 \\
\text { cigarros }\end{array}$ & 30 & 323 & 374 & 727 & \multirow{3}{*}{0,077} \\
\hline & $\begin{array}{c}\text { Menos de } 20 \\
\text { cigarros }\end{array}$ & 21 & 118 & 163 & 302 & \\
\hline & Total & 51 & 441 & 537 & 1.029 & \\
\hline \multirow{5}{*}{$\begin{array}{l}\text { Faixa de } \\
\text { idade de } \\
\text { início do } \\
\text { tabagismo }\end{array}$} & Menos de 10 anos & 5 & 32 & 59 & 96 & \multirow{5}{*}{0,510} \\
\hline & 11 a 20 anos & 47 & 406 & 477 & 930 & \\
\hline & 21 a 30 anos & 5 & 41 & 52 & 98 & \\
\hline & Mais de 30 anos & 1 & 6 & 13 & 20 & \\
\hline & Total & 58 & 485 & 601 & 1.144 & \\
\hline \multirow{4}{*}{$\begin{array}{l}\text { Escala de } \\
\text { Depressão } \\
\text { (HAD) }\end{array}$} & Improvável & 24 & 245 & 313 & 582 & \multirow{4}{*}{0,293} \\
\hline & Possível & 18 & 143 & 184 & 345 & \\
\hline & Provável & 18 & 125 & 128 & 271 & \\
\hline & Total & 60 & 513 & 625 & 1.198 & \\
\hline \multirow{4}{*}{$\begin{array}{l}\text { Escala de } \\
\text { Ansiedade } \\
\text { (HAD) }\end{array}$} & Improvável & 22 & 153 & 209 & 384 & \multirow{4}{*}{0,072} \\
\hline & Possível & 11 & 186 & 201 & 398 & \\
\hline & Provável & 26 & 175 & 217 & 418 & \\
\hline & Total & 59 & 514 & 627 & 1.200 & \\
\hline
\end{tabular}


mulheres estarem mais motivadas a parar de fumar do que os homens, embora se saiba que os homens apresentem melhores índices de cessação do que as mulheres nos programas de cessação de tabagismo. Os níveis prováveis de depressão e ansiedade também foram mais prevalente nos pacientes do gênero feminino e aqueles com menor escolaridade. Como as comorbidades psiquiátricas estâo relacionadas com piores índices de cessação, isso reforça os dados apresentados no estudo de Ferguson et al. ${ }^{11}$, no qual encontraram-se alguns preditores de sucesso de abstinência do tabagismo, tais como: gênero masculino, nível de ensino superior, maior motivação, ausência de comorbidades psiquiátricas e menos sintomas de depressão.

Lembrou-se que a relação entre tabaco e pobreza, como um ciclo vicioso, já vem sendo bem documentada. $\mathrm{Na}$ maioria dos países existe associação entre tabagismo, baixa renda e baixo nível de escolaridade. No Brasil, entre os grupos de indivíduos com baixo nível de escolaridade, a probabilidade desses se tornarem fumantes é cinco vezes maior do que indivíduos que têm o terceiro grau ${ }^{12}$. Sabe-se também que os transtornos depressivos são mais comuns em mulheres e em pessoas com menores rendas e níveis de escolaridade ${ }^{13}$. Neste estudo, além do tabagismo, os fumantes com menores renda e escolaridade também apresentaram maior nível provável de ansiedade e depressão. $\mathrm{O}$ uso do cigarro está associado com a possibilidade de prazer e o alívio de angústias, como exemplificado por Rondina et al. ${ }^{14}$ de que alguns fumantes deprimidos podem usar o tabaco para aliviar seus sentimentos negativos.

Neste trabalho, a idade de início de tabagismo confirma os dados da literatura: a maioria iniciou o tabagismo na adolescência, fase da vida em que estão presentes as influências de grupo, a rebeldia e a autoafirmação. Estes achados foram coincidentes com os estudos realizados por Levy et al..$^{15}$ e Pedersen ${ }^{16}$, no que se refere à forte associação entre o início do tabagismo na adolescência e o posterior aparecimento dos sintomas de depressão. Esses autores também encontraram forte associação entre a idade de início do tabagismo e a ansiedade. Tabagismo na adolescência parece ser um marcador para psicopatologias futuras, pois essa fase da vida é um período de maior vulnerabilidade neuronal para os efeitos da nicotina sobre a função sináptica e o desenvolvimento cerebral ${ }^{17}$.

Em relação aos dados deste estudo, observou-se uma tendência entre o nível provável de ansiedade e a idade de início do tabagismo $(p=0,057)$. Outro detalhe que chama atençáo foi a ausência de diferença significativa entre quantidade de tabaco por dia e os níveis de ansiedade e depressão, o que diverge da literatura pesquisada. Um estudo feito por Kang e $\mathrm{Lee}^{18}$ na Coreia identificou a presença de depressão em $31,3 \%$ dos fumantes de mais de dois maços por dia e, entre 18,7\% dos que fumavam, menos da metade de um maço por dia. Esse mesmo estudo mostrou índice de depressão entre os não fumantes de $17,8 \%$, o qual foi menor do que o encontrado entre aqueles que fumavam menos de meio maço ou mais de dois maços dia. Outros estudos vêm demonstrando que pacientes ansiosos e deprimidos tendem a aumentar o consumo de tabaco como automedicaçấo para minimizar os sintomas ${ }^{17-18}$.

Os pacientes com ansiedade e depressão prováveis se encontravam na faixa etária entre 41 e 50 anos. Este dado é corroborado por outro estudo, no qual a média de idade foi de 46 anos, o que pode ser também um indicativo de um momento no ciclo de vida, caracterizado por alteraçôes hormonais importantes, e, no caso especifico das mulheres, a proximidade da menopausa ${ }^{19}$.

Conforme dito anteriormente, a metade dos fumantes desta amostra apresentou grau elevado de motivação, independentemente dos níveis de ansiedade e depressão, mas quando comparam-se as variáveis deste estudo com o grau de motivação nos três níveis da Escala de Richmond encontram-se algumas diferenças a serem consideradas, tais como: o nível de motivação moderada está presente em $84 \%$ nos fumantes que iniciaram o tabagismo entre 11 a 20 anos, em $41 \%$ dos fumantes com apenas o ensino fundamental, e em $73, \%$ dos que fumam até 20 cigarros por dia. Foram encontrados níveis baixos de motivação em $44 \%$ dos pacientes com nível de ansiedade provável e 30\% com nível provável de depressão. Em estudo realizado por Melo et al. ${ }^{20}$, comparando os estágios motivacionais com ansiedade e depressão, chama atenção o fato de que quanto maiores os escores de ansiedade e de depressão menor a motivação e aderência ao tratamento. Nossos resultados não foram consistentes com este estudo, uma vez que não se encontrou correlaçáo entre motivaçáo elevada e os níveis

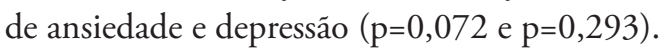

$\mathrm{O}$ fato de os pacientes pesquisados estarem buscando uma vaga para tratamento em um dos Centros de Referência pode ter influenciado nas informaçóes relacionadas à motivaçáo e isto pode ser considerado um fator de confusão para essa variável. Outra limitação deste estudo é que, quando se aplica um teste, ele afere apenas aquele momento em que a pessoa está vivendo, e pode estar sob influência de situações e fatos transitórios.

\section{CONCLUSÃO}

Os dados do estudo sugerem que cerca de $30 \%$ dos fumantes que buscaram atendimento nos Centros de Referência do Distrito Federal apresentaram níveis prováveis de ansiedade e depressão. Destes, 50\% estavam 
altamente motivados para deixar de fumar. $\mathrm{O}$ gênero feminino apresentou os maiores índices prováveis de ansiedade e depressão; no entanto, se destaca em relação ao gênero masculino no que se refere à motivaçáo para cessação.

A partir desses resultados, chama-se atenção para a necessidade de se avaliar a motivação dos pacientes e o preparo das equipes de atendimento quanto ao reconhecimento prévio das comorbidades psiquiátricas, por meio da detecção precoce de sinais e sintomas, o que pode definir o tratamento do tabagismo mais adequado, elevando, assim, as taxas de sucesso.

\section{AGRADECIMENTOS}

Ao Dr. Celso Antônio Rodrigues da Silva, pelo incentivo para a realização deste trabalho, pelo seu entusiasmo contagiante à frente do Programa de Controle do Tabagismo no Distrito Federal.

\section{CONTRIBUIÇÕES}

Maria Suélita de Lima contribuiu na obtenção análise e interpretação dos dados; Carlos Alberto de Assis Viegas contribuiu na redação e revisão final.

\section{Declaração de Conflito de Interesses: Nada a Declarar.}

\section{REFERÊNCIAS}

1. Instituto Brasileiro de Geografia e Estatística. Pesquisa nacional por amostra de domicílios: tabagismo 2008. Rio de Janeiro; 2009.

2. Zvolensky MJ, Stewart SH, Vujanovic AA, Dubravka G, Steeves D. Anxiety sensitivity and anxiety and depressive symptoms in the prediction of early smoking lapse and relapse during smoking cessation treatment. Nicotine Tob Res. 2009;11(3):323-31.

3. Williams JM, Ziedonis D. Addressing tobacco among individuals with a mental illness or an addiction. Addict Behav. 2004 Aug;29(6):1067-83.

4. Lawrence D, Mitrou F, Zubrick SR. Smoking and mental illness: results from population surveys in Australia and the United States. BMC Public Health. 2009 Aug;9:285.

5. Miller WR, Rollnick S. Entrevista motivacional: preparando as pessoas para a mudança de comportamentos adictivos. Porto Alegre: Artmed; 2001.

6. Zigmond AS, Snaith RP. The hospital anxiety and depression scale. Acta Psychiatr Scand. 1983;67:361-70.

7. Marcolino JAM, Mathias LAST, Piccinini Filho L, Guaratini AA, Suzuki FM, Alli LAC. Escala hospitalar de ansiedade e depressão: estudo da validade de critério e da confiabilidade com pacientes no pré-operatório. Rev Bras Anestesiol. 2007;57(1):52-62.

8. Richmond R, Kehoe L, Webster I. Multivariate models for predicting abstention following intervention to stop smoking by general practioners. Addiction. 1993;88: 1127-35.

9. McClave AK, Dube SR, Strine TW, Kroenke K, Caraballo RS, Mokdad AH. Associations between smoking cessation and anxiety and depression among U.S. adults. Addict Behav. 2009 Jun-Jul;34(6-7):491-7.

10. Issa JS, Peres GH, Diament J, Zavattieri AG, Oliveira KU. Efetividade da bupropiona no tratamento de pacientes tabagistas com doenças cardiovascular. Arq Bras Cardiol. 2007;88(4):434-40.

11. Ferguson JA, Patten CA, Schroeder DA, Offord KP, Eberman KM, Hurt RD. Predictors of 6-month tobacco abstinence among 1224 cigarette smokers treated for nicotine dependence. Addict Behav. 2003;28:1203-18.

12. Organização Pan-Americana da Saúde. Tabaco e pobreza, um círculo vicioso: a convenção quadro de controle do tabaco: uma resposta. Brasília: Ministério da Saúde; 2004.

13. Lima MS. Epidemiologia e impacto social. Rev Bras Psiquiatr. 1999;21 supl 1: SI1-5.

14. Rondina RC, Gorayeb RS, Botelho C. A dinâmica psicológica do tabagismo: o papel de características de personalidade, psicopatologia, fatores genéticos e neurobiológicos no comportamento de fumar tabaco. Cuiabá: Entrelinhas; 2004.

15. Levy AS, Anna LM, Westein BS, Reamy SB, Reyner CJ, Syed T, et al. Comunication about smoking between depressed adolescents and their parents. Nicotine Tob Res. 2010 Mar;12(3):191-7.

16. Pedersen W, Soest T. Smoking, nicotine dependence and mental health among young adults: a 13- year population-based longitudinal study. Addiction. 2009 Jan;104(1):129-37.

17. Lemos T, Gigliotti AP. Transtornos psiquiátricos e tabagismo. In: Viegas CAA, coord. Tabagismo do diagnóstico à saúde pública. São Paulo: Atheneu; c2007. p. 185-96.

18. Kang E, Lee J. A longitudinal study on the causal association between smoking and depression. J Prev Med Public Health. 2010 May;43(3):193-204.

19. Calheiros PRV, Oliveira MS, Wagner MF, Matos KS. Sintomas de ansiedade em tabagistas no início do tratamento. Revista de Psicologia da IMED. 2009;1(1):46-55.

20. Melo WV, Oliveira MS, Ferreira EA. Estágios motivacionais, sintomas de ansiedade e de depressão no tratamento do tabagismo. Interação Psicol. 2006;10(1): 91-9. 


\section{Abstract}

Introduction: Anxiety, depression and low motivation can interfere with successful treatment of smoking. Objective: To evaluate the level of anxiety, depression and motivation of smokers enrolled in cessation programs in the Federal District. Method: A cross-sectional study involving 1,233 smokers enrolled at 19 Reference Centers. Instruments: Hospital Anxiety and Depression Scale, motivation (Richmond test), and forms with social and demographic data. For data analysis, where appropriate, the Student t test, chi-square and Spearman correlation were used. Results: Women had higher level of anxiety $(\mathrm{p}<0.01)$, depression $(\mathrm{p}<0.01)$, and motivation $(\mathrm{p}<0.01)$ for smoking cessation when compared with men. There was no difference between motivation and socio-demographic variables $(p>0.05)$. Low income and low education levels were associated with anxiety $(\mathrm{p}<0.01$ and $\mathrm{p}<0.03)$ and depression $(\mathrm{p}<0.01$ and $\mathrm{p}<0.04)$ levels. Age of smoking initiation was associated with level of depression, the younger the onset of smoking, the greater likelihood of depression $(\mathrm{p}<0.02)$. There was no correlation between number of cigarettes per day and level of motivation to quit smoking, and levels of anxiety and depression. The motivation was not associated with anxiety and depression. Conclusion: There was association regarding levels of anxiety, depression and smoking. About 30\% of smokers presented likely levels of anxiety and depression, among those 50\% had high motivation to quit smoking, especially women. Those results indicate the importance of assessing these symptoms and motivation at the beginning of treatment to raise the rates of cessation.

Key words: Tobacco Use Cessation; Anxiety; Depression; Motivation

\section{Resumen}

Introducción: La ansiedad, la depresión y la baja motivación pueden interferir con el éxito del tratamiento del tabaquismo. Objetivo: Evaluar el grado de la ansiedad, la depresión y la motivación de los fumadores atendidos en el Distrito Federal. Método: Estudio transversal que incluyó a 1.233 fumadores atendidos en 19 Centros de Referencia. Instrumentos utilizados: Hospital Anxiety and Depression Scale, test de motivación de Richmond y cuestionario de datos socio-demográficos. Para el análisis de los datos se utilizó, en prueba de su caso, el test de t de student, chicuadrado y correlación de Spearman. Resultados: Las mujeres tuvieron una mayor ansiedad $(\mathrm{p}<0,01)$, depresión $(\mathrm{p}<0,01)$, y un mayor grado de motivación $(\mathrm{p}<0,01)$ para dejar de fumar en comparación con los hombres. No hubo diferencias entre la correlación de la motivación y las variables socio-demográficas ( $p>0,05)$. Bajos niveles de educación $y$ de ingresos bajos se asociaron con la ansiedad $(\mathrm{p}<0,01 \mathrm{y} p<0,03)$ y depresión $(\mathrm{p}<0,01 \mathrm{y} p<0,04)$. La edad de inicio del consumo del tabaco se asoció con el nivel de depresión, cuánto más joven es el inicio del acto de fumar, mayor el riesgo de depresión $(\mathrm{p}<0,02)$. No hubo correlación entre el número de cigarrillos por día y el nivel de motivación para dejar de fumar, con niveles de ansiedad y depresión. La motivación no se asoció con la ansiedad y la depresión. Conclusión: En este estudio hubo una asociación entre los niveles de ansiedad, la depresión y el tabaquismo. Alrededor del 30\% de los fumadores tenían probables niveles más altos de ansiedad y depresión, de los cuales 50\% tenían una alta motivación para dejar de fumar, especialmente las mujeres. Estos resultados destacan la importancia de evaluar síntomas de depresión/ansiedad y la motivación en el inicio del tratamiento para elevar las tasas de cesación.

Palabras clave: Cese del Uso de Tabaco; Ansiedad; Depresión; Motivación 